\title{
A Case Report: In-hospital acute papillary muscle rupture in SARS-COV 2 positive patient late after acute inferior myocardial infarction
}

Tarique chachar ${ }^{1}$, Vinayak Vadgaonkar², Zaid Arekat ${ }^{2}$, Fuad Abdul qader ${ }^{2}$, and Seham Ali Abdulrahman ${ }^{2}$

${ }^{1}$ Bahrain Defence Force

${ }^{2}$ Bahrain Defense Force Hospital

November 25, 2020

\begin{abstract}
Papillary muscle rupture is one of the rare and potentially life-threatening complication usually following acute myocardial infarction (AMI). Acute papillary muscle rupture (PMR) results in severe mitral regurgitation rapidly progressing to florid pulmonary edema and cardiogenic shock. Without emergent surgical intervention, the mortality is extremely high. The management of STEMI patients in COVID 19 pandemic is difficult and challenging. We present an unusual case of 56 years old male who presented with acute inferior wall myocardial infarction (IWMI), underwent successful thrombolysis, developed reinfarction 5 days later complicated by acute postero-medial papillary muscle rupture (PPMR) leading to severe acute mitral regurgitation.
\end{abstract}

\section{CASE REPORT}

A Case Report: In-hospital acute papillary muscle rupture in SARS-COV 2 positive patient late after acute inferior myocardial infarction

Tarique S. chachar MD*1, Vinayak Vadgaonkar MD ${ }^{1}$, Zaied Arekat FRSCSC ${ }^{1}$, Fuad AbdulQader ${ }^{1}$, Seham A. Saif ABIM, SBIM**1

*First Author

${ }^{* *}$ Corresponding Author

1 Affiliation - MOHAMMED BIN KHALIFA BIN SALMAN ALKHALIFA CARDIAC CENTER- KINGDOM OF BAHRAIN

\section{Abstract}

Papillary muscle rupture is one of the rare and potentially life-threatening

complication usually following acute myocardial infarction (AMI). Acute papillary muscle rupture (PMR) results in severe mitral regurgitation rapidly progressing to florid pulmonary edema and cardiogenic shock. Without emergent surgical intervention, the mortality is extremely high. The management of STEMI patients in COVID 19 pandemic is difficult and challenging. We present an unusual case of 56 years old male who presented with acute inferior wall myocardial infarction (IWMI), underwent successful thrombolysis, developed reinfarction 5 days later complicated by acute postero-medial papillary muscle rupture (PPMR) leading to severe acute mitral regurgitation. 


\section{Introduction}

Papillary muscle rupture is one of the rare and potentially life-threatening

complication usually following acute myocardial infarction (AMI). Acute and complete papillary muscle rupture (PMR) results in severe mitral regurgitation rapidly progressing to florid pulmonary edema and cardiogenic shock. ${ }^{1}$ Without emergent surgical intervention, the mortality is extremely high.

We present an unusual case of 56 years old male who presented with acute

inferior wall myocardial infarction (IWMI) and underwent successful thrombolysis but unfortunately, developed reinfarction 5 days later complicated by acute postero-medial papillary muscle rupture (PPMR) leading to severe acute mitral regurgitation. He was promptly diagnosed and underwent emergent mitral valve replacement.

Key words: COVID - 19, Mitral regurgitation, papillary muscle rupture, Acute myocardial infarction.

\section{CASE PRESENTATION}

A 56-year-old smoker not known to have any medical illness presented to us with progressive chest pain of one-hour duration. Initial electrocardiogram

(ECG) showed inferior leads ST segment elevation (STE) with reciprocal changes in anterior leads (figure 1A) and ST elevation (STE) in right V4R suggestive of acute inferior and right ventricular current of injury. His systemic examination was unremarkable. Peak highly sensitive troponin I assay was $>25$ (Normal range $<0.010$ ) and creatine kinase (CK) level 4764 (Normal range $20-200 \mathrm{IU} / \mathrm{L}$ ).

Based on our institutional protocol in this current covid-19 era, he underwent thrombolysis with Tenecteplase. His event was complicated with transient hypotension and heart block which resolved spontaneously. He had successful thrombolysis with significant resolution of STE (figure 1B) and chest pain free 60 minutes post administration of Tenecteplase.

He was managed conservatively on dual anti-platelet therapy and statin. His GeneXpert test for SARS COV 2 turned out to be positive without any significant constitutional symptoms. He was admitted to COVID 19 intensive care unit with full cardiac monitoring.

A B 

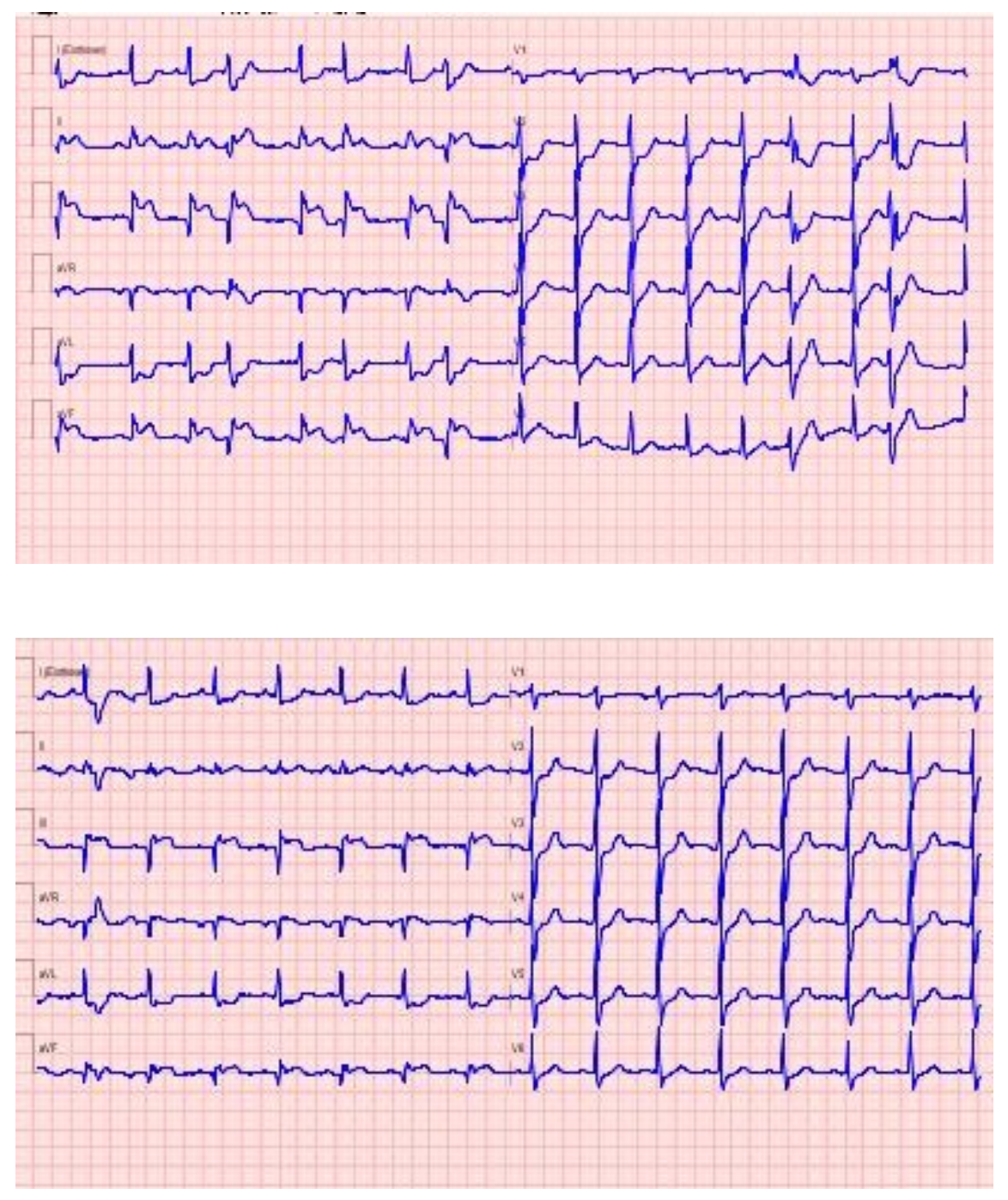

Figure 1: A- Pre-thrombolysis B- Post-thrombolysis

Transthoracic echocardiography (TTE) revealed akinetic base to mid inferior, inferolateral and lateral walls. Left ventricular ejection fraction (LVEF) was visually estimated to be $45 \%$. Mild functional mitral regurgitation with insignificant pulmonary hypertension. Normal right ventricular systolic function.

His further stay was uneventful with no symptoms of angina, shortness of breath and constitutional symptoms. He was ambulated from second day onwards.

On $5^{\text {th }}$ day he developed severe acute chest pain with diaphoresis. His recorded blood pressure was $70 \mathrm{mmhg}$ systolic, heart rate of 145/minute and 
pulse oximetry saturation $70 \%$ on room air. On auscultation, He did not have any audible murmur but had extensive rales extending till mid-zones of both lung fields. ECG revealed STE in leads II, III and AVF with reciprocal changes anteriorly (figure3) suggesting inferior wall re-infarction.

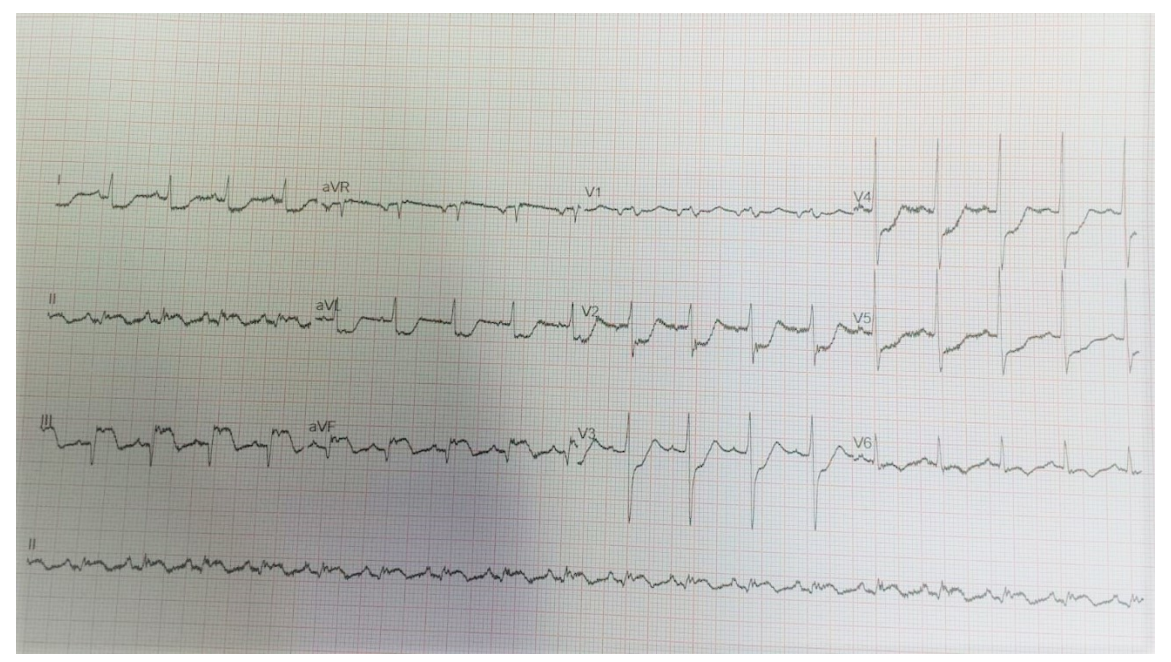

Figure 2: $5^{\text {th }}$ day post-thrombolysis in Covid ICU

Patient underwent immediate mechanical intubation and started on inotropes.

In view of patient's cardiogenic shock, he was emergently taken to cardiac catheterization laboratory.

Coronary angiogram (CAG) via right radial artery showed subtotal occlusion of proximal right coronary artery (RCA) with thrombolysis in myocardial infarction grade I flow (TIMI I flow), his left system did not show any significant lesion. Proximal RCA was stented with TIMI III flow. He continued to be in pulmonary edema with high inotropic supports throughout the procedure. 


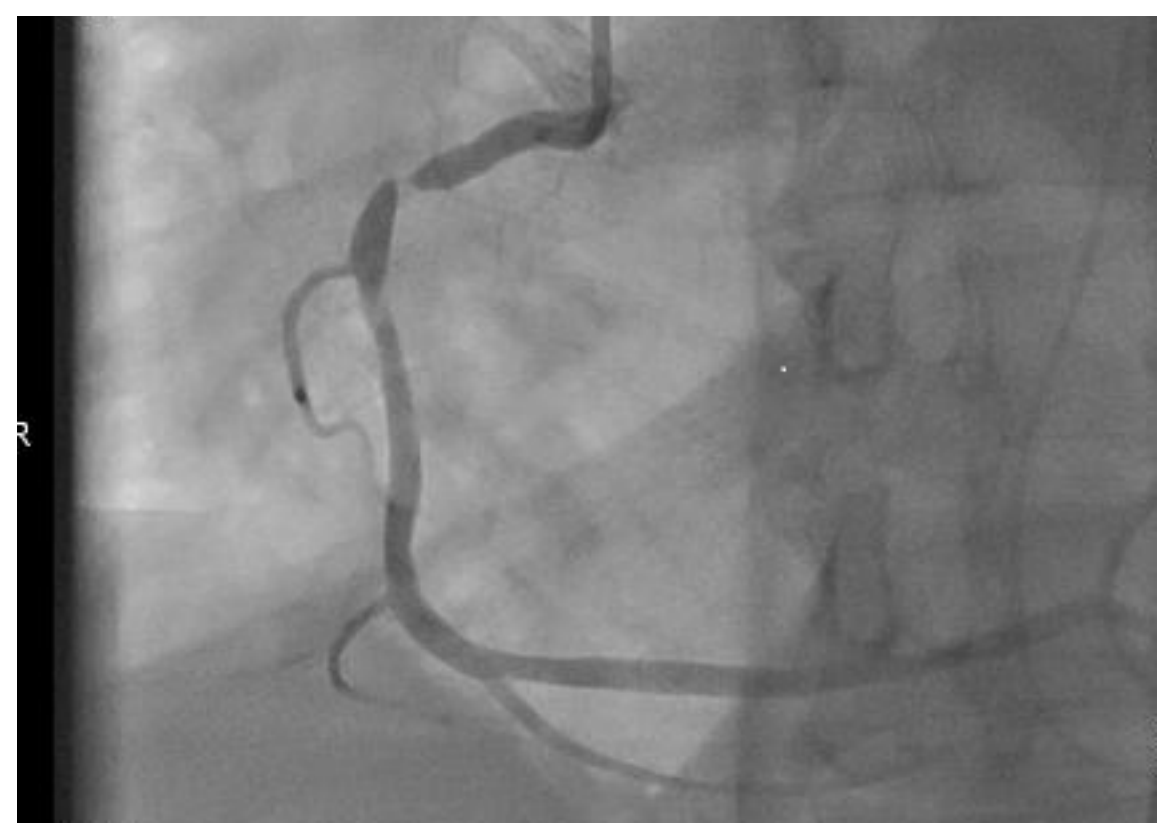

Figure 3: Coronary angiography - critical stenosis of right coronary artery

Transthoracic echocardiography performed immediately after CAG showed hyperdynamic left ventricle with severe hypokinesis of inferior and inferolateral wall. There was chaotic movement of rounded mass in left ventricle with complete flail of anterior mitral leaflet (figure 4a). The mass did not prolapse into left atrium. Short axis view confirmed the mass to be ruptured posteromedial papillary muscle (PMPM) (figure 4b) causing severe acute eccentric posterolateral directed jet of Mitral regurgitation into nondilated left atrium (4c, d). It was difficult to establish whether it was partial or complete rupture of PPM considering suboptimal TTE study as it was performed in hemodynamically unstable patient with respiratory distress.

A B 

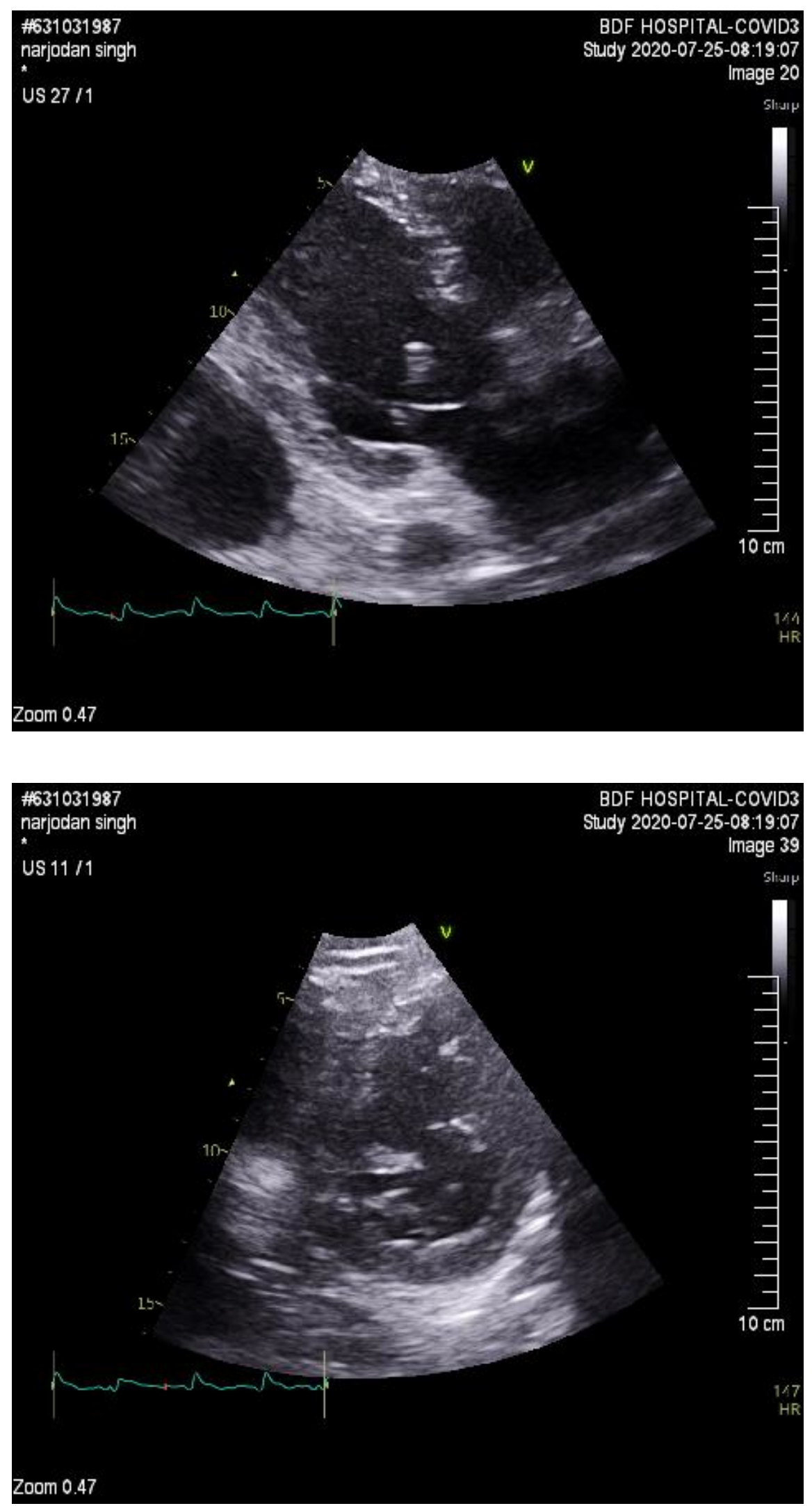

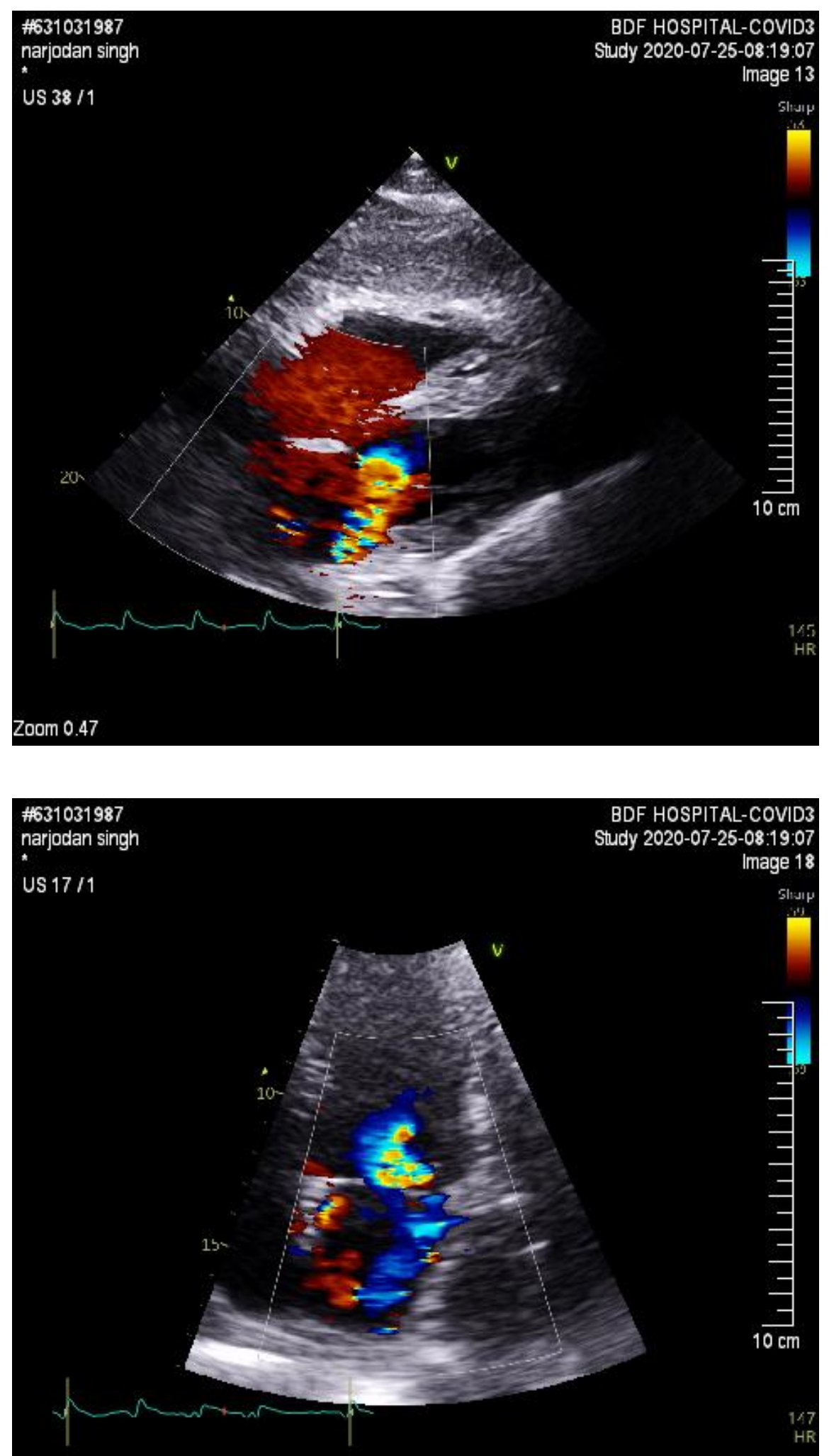

BDF HOSPITAL-COVID3 Study 2020-07-25-08:19:07 Image 18 जॉเा1

\section{Zoom 0.47}




\section{D}

Figure 4: Transthoracic echocardiography. A- D

Intra-aortic balloon pump was secured for further stabilization and multi-

disciplinary team consensus was for urgent mitral valve intervention.

He was subsequently taken to operation theater on full personal protective

equipment (PPE). On surgical inspection mitral valve anatomy was unsuitable

for repair and hence decision was taken for emergent mitral valve

replacement. Intra-operative findings showed flail and unsupported anterior

mitral valve leaflet due to complete rupture of PMPM head. Mitral valve

replacement was performed using $31 \mathrm{~mm}$ magna ease bioprosthetic valve.

The patient was weaned off from IABP and then from inotropes. The

hemodynamics improved post operatively. He was further managed

medically.

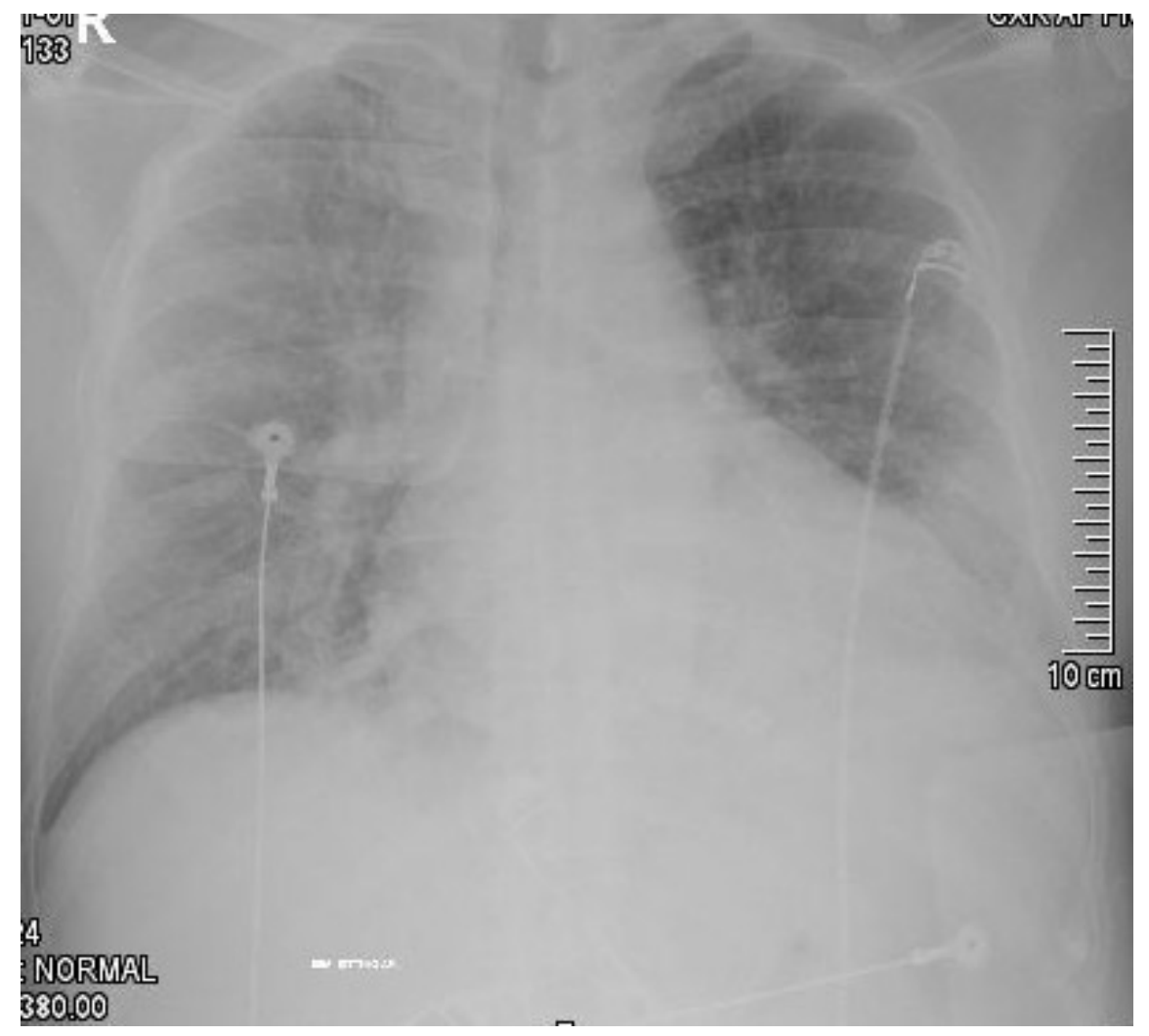

Figure 5: CHEST XRAY WITH PULMONARY CONGESTION

DISCUSSION

Primary percutaneous intervention (pPCI) has revolutionized the treatment in ST-elevation myocardial infarction (STEMI) and tremendously decreased the rate of mechanical complications. In the pre-fibrinolytic 
period, the rate of mechanical complications was $6 \%$, while it has been reduced to $<0.5 \%$ in the revascularization era. In an analysis of 4 million hospitalized STEMI patients from 2003 to 2015, mechanical complications have been reported in only $0.27 \%$ of patients with PMR incidence as low as $0.05 \%{ }^{2}$

The optimal timing of papillary muscle rupture is 2 to 7 days post-myocardial infarction. Mortality was reported $50 \%$ within 24 hrs without surgical intervention. $82 \%$ of patients with first myocardial infarction were reported to have papillary muscle rupture. ${ }^{3,4}$

Echocardiography with Doppler is the cornerstone for the diagnosis of this fatal complication. The sensitivity of TTE to visualize the structural abnormalities has been estimated to be $65-85 \%$, with transesophageal echo sensitivity approaching $92-100 \%$.

The rupture of the posteromedial papillary muscle is 6 to 12 times more common than the anterolateral papillary muscle due to its single blood supply from the posterior descending artery of the dominant right coronary artery (RCA) or dominant left circumflex artery (LCX), causing inferior wall myocardial infarction (IWMI). ${ }^{5}$ Most cases of PMR occur after small areas of ischemia, usually less than $25 \%$ of left ventricular with poor collaterals, and is thought to be due to preserved ventricular function exhibiting increased shear stress to the ischemic papillary muscle.

Our patient did not have an audible murmur of severe mitral regurgitation, which could be explained by the rapid equalization of pressures within the left ventricle and left atrium.

This case was unusual as he had successful thrombolytic reperfusion of acute inferior infarction at presentation. Later he again developed in-hospital re-infarction leading to PMR. Whether it is related to covid 19 infections is unknown to us with a lack of strong evidence.

There have been reports of increased coronary artery thrombus burden in patients with STEMI in COVID 19 positive patients. ${ }^{6}$ This is consistent with an increased frequency of thrombotic strokes, particularly in young people, during the pandemic. Alterations in the coagulation system, abnormal platelet function, or abnormal endothelial function have been postulated. ${ }^{7}$

Early diagnosis, prompt hemodynamic support to reduce afterload with appropriate medications as well as with devices like intra-aortic balloon pump (IABP), extracorporeal membrane oxygenation (ECMO), and Tandem heart, and immediate surgical intervention are required to reduce morbidity and mortality due to papillary muscle rupture secondary to Acute myocardial infarction. ${ }^{8}$

References

1. Kutty RS, Jones N, Moorjani N. Mechanical complications of acute myocardial infarction. Cardiol Clin. 2013; 31:519-31. doi: 10.1016/j.ccl.2013.07.004. [PubMed] [Google Scholar]

2. Elbadawi A., Elgendy I.Y., Mahmoud K. Temporal trends and outcomes of mechanical complications in patients with acute myocardial infarction. J Am Coll Cardiol Intv. 2019; 12:1825-1836. doi: 10.1016/j.jcin.2019.04.039. [Google Scholar]

3. Nguyen S, Umana-Pizano JB, Donepudi R, Dhoble A, Nguyen TC. Minimally Invasive Mitral Valve Repair for Acute Papillary Muscle Rupture During Pregnancy. Ann. Thorac. Surg. 2019 Feb;107(2): e93-e95. doi: 10.1016/j.athoracsur.2018.06.048. [PubMed]

4. Harari R, Bansal P, Yatskar L, Rubinstein D, Silbiger JJ. Papillary muscle rupture following acute myocardial infarction: Anatomic, echocardiographic, and surgical insights. Echocardiography. 2017 Nov;34(11):1702-1707.

https://doi.org/10.1111/echo.13739 [PubMed]

5. Kunkel K, Anwaruddin S. Papillary muscle rupture due to delayed STEMI presentation in a patient self-isolating for presumed COVID-19. JACC Case Reports. 2020 Aug;10(2):1633-1636. https://doi.org/10.1016/j.jaccas.2020.06.036 
6. Roffi M, Guagliumi G, Ibanez B. The Obstacle Course of Reperfusion for ST-SegmentElevation Myocardial Infarction in the COVID-19 Pandemic. Circulation 2020; 141:1951. doi.org/10.1161/CIRCULATIONAHA.120.047523 7. Varga Z, Flammer AJ, Steiger P, et al. Endothelial cell infection and endotheliitis in COVID-19. Lancet 2020; 395:1417. Doi.org/10.1016/S0140-6736(20)30937-5

8. DiVita M, Visveswaran G.K, Makam K. Emergent TandemHeart-ECMO for acute severe mitral regurgitation with cardiogenic shock and hypoxaemia: a case series. Eur Heart J Case Rep. 2020; 4:1-6. doi: 10.1093/ehjcr/ytz234 [PMC free article] [PubMed] [Google Scholar] 